hospice-led LTV service initially by specialist nurse roles, evolving further with the development of a novel senior care assistant role (SCA).

The LTV service From April 2020 to February 2021 the LTV service supported 68 children and young people with level 1 , 2 or 3 ventilation needs, children and young people with a tracheostomy and those with complex respiratory care needs. The service supports discharge planning, coordinates care outside of hospital, provides specialist clinical education for families and professionals and has supported care at end of life for LTV children and young people. Care is overseen by two clinical nurse specialists (CNSs), two LTV nurses and two SCA. Full theoretical and practical training is in place with education cascaded by specialist team and tailored to individual hospice staff needs.

SCA role development The development of the SCA role was perceived as innovative and cost effective way to support the CNS in delivery of LTV care as the caseload and complexity of LTV children and young people developed. From April 2020 to February 2021, SCAs spent 69\% of their time on clinical tasks, $17 \%$ teaching and $8 \%$ on practice development. Key elements of the SCA role (with CNS oversight) are:

- Teaching/training/mentoring nurses and care assistants. Training and supporting families.

- Developing child specific portfolios.

- Advocating for children and young people in MDTs and professional liaison.

- Sourcing equipment.

- Assessment of stable and unstable children and young people, planning and evaluating care.

Conclusion Developing this innovative SCA role has ensured patients receive increased professional contact, enabled their own personal professional development and makes best use of the organisation's available workforce in a cost effective way. The SCA acts as role model, demonstrating that safe and effective care is possible with the introduction of a skill mix for children and young people with LTV needs in the region.

\section{P-109 LITTLE HAVENS CHILDREN'S HOSPICE STAY AND PLAY GROUP}

Evie Bocking, Lucy Smith. Havens Hospices, Southend-on-Sea, UK

\subsection{6/spcare-2021-Hospice.126}

Introduction Parents using the children's hospice fed back that they would like to access a play group where they can meet other families in similar situations and feel safe and cared for with their children. We introduced a 'stay and play' group to meet the identified need and improve access for children with additional needs and improve service provision.

\section{Aims}

- Reduce inequality in access to play groups for children with different needs.

- Provide a play group for under $4 \mathrm{~s}$ for children who have different needs.

- Improve parents' ability to adapt and play with their children.

- Help children meet their potential through play.

- Enable parents to meet others in similar situations and build their own network.
- Enhance parent and carer confidence and resilience, transferable to the community setting.

- Introduce families and children to the hospice through a less daunting approach.

Method

- Whole case load reviewed with a newly introduced holistic assessment.

- Parents with children under 4 identified they were not accessing play groups because their child's needs could not be met.

- Parents identified as socially isolated.

- 26 families were identified as having a child under 4 from the patient administration system.

- Parents were invited to attend the 'stay and play' group.

- At four weeks a questionnaire was administered with parents to evaluate the group.

- Four weekly questionnaires are planned.

Results

- At four weeks the 'stay and play' group was positively evaluated.

- Families feel safe.

- Families can talk to other families who understand their child's needs.

- Decision to continue with the group and continue to evaluate regularly.

Conclusion This is a safe space for families to attend, allow their child to play and feel supported and understood. Plans to extend the age range to five and under and develop this concept further.

\section{P-110 DEVELOPING A CHILDREN'S HOSPICE COMMUNITY SERVICE DURING THE COVID-19 PANDEMIC}

Janet Miller. Trinity Hospice, Bispham, UK

10.1136/spcare-2021-Hospice. 127

Brian House children's hospice on the Fylde coast in the North West of England provides palliative and end-of-life care for children and families with complex needs and lifelimiting conditions. A recent research study, although ongoing, identified that some families were not accessing a service that was available to them in their local area. The team developed an action plan to build a community service enabling palliative and end-of-life care to be delivered in the community, therefore reaching out to the families identified in the study. COVID-19 implemented restrictions that impacted on the respite available to families due to reduced capacity; we had to react to this. COVID-19 empowered the team time to reassess the community service action plan ensuring a service was delivered in line with government guidelines and restrictions.

A proposal of a community service was presented to parents, their initial reaction was hesitance. Despite parents' hesitation Brian House launched the community service. Our community team reacted to the pandemic successfully as more and more families came on board with this different way of accessing care provision. We networked and created links that are invaluable and crucial to developing a community service that would meet the needs of our children and families. 
There have been many challenges along the way, however, our journey continues and we have many more miles to go. Early feedback from parents and families is positive and they are enjoying the diversity of our service.

\section{P-111 BEYOND THE CLINICAL - HOW WELL DO WE REALLY MEET THE NEEDS OF OUR FAMILIES?}

Vanessa Spinks, Lynn Grayson, John Pye, Siobhan McCoy. Derian House Children's Hospice, Chorley, Lancashire

\subsection{6/spcare-2021-Hospice.128}

Background As practitioners and clinicians who work closely with children, young people and their families, we always assume we know what the families need from hospices.

Aim(s) To review the current service offering of the hospice, to identify gaps in provision, to highlight any barriers there are to accessing services and to shape our delivery to meet the articulated future needs of our families and our commissioners.

Methods We commissioned an independent research company to undertake the research project which included a desktop literature review, quantitative and qualitative interviews with 100 families and interviews with stakeholders across the North West including FOI requests to CCGs. Neonatal and bereaved families were not included in this study.

Results Our families identified respite as the most important service offered by the hospice with wellbeing services identified as the second most important. Our stakeholders and CCGs acknowledged funding for palliative care is patchy across the North West and were concerned whether Derian House can continue to offer the services we currently provide. Additionally, the research also highlighted a big gap in transition services between children's and adults' hospices.. The results of the research have informed the strategy for the hospice for the next three years and CCGs will be using the research to revise and develop their own services for children and young people.

Conclusions The results supported our continued provision of planned respite, it also highlighted areas which may act as barriers to accessing services which include transport and also the information provided to external services explaining the facilities offered to families. These barriers identified by this research project now form part of our strategy over the next three years. We will continue to review services and engagement with families, and will be further exploring needs for neonatal families and the need of families for end-of-life and bereavement.

\section{P-112 PARENT LIVED EXPERIENCES OF UNMET NEEDS, ACCESS AND EXPECTATIONS IN CARE FOR LIFE-LIMITED CHILDREN}

1,2,3 Georgina Constantinou, ${ }^{2}$ Gurch Randhawa, ${ }^{2}$ Erica Cook, ${ }^{3}$ Elaine Tolliday. ${ }^{1}$ City, University of London, London, UK; ${ }^{2}$ University of Bedfordshire, Luton, UK; ${ }^{3}$ Keech Hospice Care, Luton, UK

\subsection{6/spcare-2021-Hospice.129}

Background Harnessing the experiences of parents of life-limited children remains a research priority nationally and internationally. Existing evidence outlines the needs of this group, however, less information is available as to how well these needs are being met by services from the perspective of parents in the UK. The parent responsibility for providing and navigating care for their child is well documented, therefore, it is important we understand the barriers and facilitators faced by families when accessing services for their children.

Aim To examine how children's palliative care services can be improved to meet the needs of children and their families.

Methods A two-part qualitative study underpinned by a social constructivist and phenomenological research approach was employed. Phase 1 conducted an interview-diary study over a two-month period with parents currently caring for a life-limited child $(n=12)$ and Phase 2 carried out semi-structured interviews with bereaved parents of life-limited children $(n=5)$. The data was transcribed verbatim and thematically analysed.

Findings The findings revealed concerns with 'Communication and Information sharing', identified 'Factors Influencing access' i.e., limited funding, staffing availability, delays in services and poor perceptions of palliative care and emphasised the 'Continuous caring and Fighting' involved in being a parent of a child with a life-limiting illness.

Conclusion The parents' lived experiences, documented in this research, provide insight into the unmet needs, barriers, and facilitators to access and understanding of the role of parent expectations in care. These findings indicate that further research is warranted identifying parents' awareness of available information and resources, further investigation into the working practices between services within the children's palliative care pathway and an overall need for suitable respite to be available to families.

\section{P-113 NEW KIDS ON THE BLOCK IN PALLIATIVE CARE}

Fiona Wylie, Sheonad Laidlaw. The Prince and Princess of Wales Hospice, Glasgow, UK

\subsection{6/spcare-2021-Hospice. 130}

Background Advances in medicine means many young adults (YA) in Scotland with a life-limiting condition are living into adulthood (Fraser, Jarvis, Moran, et al., 2015; McLaughlin \& Robb, 2018; McLaughlin, Marosi, Robb, 2020). However, despite this growing trend, access to adult palliative care services is variable (Scottish University Insight Institute, 2019) with reluctance from YAs and professionals to engage (Lidstone, 2013). We explored and evaluated staff experience in caring for a YA within an adult palliative setting.

Aim(s) To explore and evaluate the lived experience of the inpatient unit team caring for a YA during an admission.

Methods Following a young adult admission all staff involved were invited to attend a facilitated reflective discussion to explore their thoughts, feelings and experiences. Questions for reflection were sent in advance of the meeting.

Results Despite the misconceptions pre-admission, the lived experience of physically caring for a young adult offered an entirely different lens and demonstrated the power of human contact in establishing rapport and trust. The admission also afforded insight into the importance of acknowledging the young adult as an expert in their own care and that when staff negotiated and accommodated this the difference observed was:

'I could really feel that the young adult felt physically and emotionally safe.' 